\title{
So...why do you want to become an anesthesiologist?
}

\author{
Colin J. L. McCartney, PhD
}

Received: 6 June 2015/ Accepted: 12 June 2015 / Published online: 23 June 2015

(C) Canadian Anesthesiologists' Society 2015

"Choose a job that you love and you will never have to work a day in your life"-Confucius

Each year during the Canadian Residency Matching Service application and selection process for anesthesia training positions, medical students are asked the familiar question, "Why do you want to be an anesthesiologist?" This could be a difficult question to answer, as many medical students will have had only brief exposure to clinical anesthesia, and most applicants will not appreciate what the specialty is really like in practice.

In this issue of the Journal, several articles examine this subject. Khan et al., ${ }^{1}$ and O'Leary and Murphy ${ }^{2}$ in their accompanying editorial, determine the factors that influence career choices in anesthesia, including finding an area that engages the "spirit" and having the right mentor (whether by traditional model ${ }^{3}$ or peer-peer mentorship). ${ }^{\mathrm{A}}$ Given the recognized importance of mentorship, Zakus et al. ${ }^{4}$ take this subject a step further by evaluating whether our anesthesia residency programs provide formal mentorship opportunities. They find, possibly unsurprisingly, that the provision of a formal mentorship program is associated with a greater likelihood of receiving support. For most students, the major factors influencing their choice of anesthesia as a career include the challenge of managing critically ill patients, the clinical

C. J. L. McCartney, PhD ( $₫)$

Department of Anesthesia, The Ottawa Hospital, The Ottawa

Hospital Research Institute, B311, Ottawa Hospital, Civic

Campus, Ottawa, ON, Canada

e-mail: cmccartney@toh.on.ca

URL: http://www.colinjlmccartney.com

C. J. L. McCartney, PhD

Faculty of Medicine, The University of Ottawa, Ottawa, ON, Canada application of physiology and pharmacology, the "handson" nature of the specialty, as well as the impact of positive role models. ${ }^{5-7}$

In my own experience, important factors in my decisionmaking were the role models who gave their time to show me the special talents that anesthesiologists possess. After becoming a physician, I spent my first year in both the medical and surgical wards of two large community hospitals where I had direct responsibility for many acutely ill patients and those with uncontrolled pain. It became obvious to me through management of these patients that the anesthesiologist was often called upon to manage critical situations where others had failed. I was inspired not only by the skill and compassion of these colleagues but also by their patience and composure. By the end of my first year, I had decided that I wanted to be an anesthesiologist, and it was the impact of these inspirational future colleagues that brought me into this specialty. Many of my colleagues have had similar experiences $^{6}$; however, like many senior medical students, when I chose anesthesia as my specialty, I had a limited idea of what the profession was really like in practice.

So now, 22 years later and as head of one of the larger departments of anesthesia in Canada, I think I finally understand what it means to be an anesthesiologist!

Anesthesia is a very short name for one of the most holistic medical specialties with exposure to almost all clinical areas in a hospital setting, including the obvious perioperative functions. Furthermore, the specialty also incorporates specific expertise in the management of critically ill patients and those with intractable acute, chronic, and often cancer-related pain. In my decades of

\footnotetext{
A https://www.sluckettg.wordpress.com/2015/05/28/peer-mentorshipor-who-am-i-to-tell-you-what-to-do/.
} 
training and practice, I have had the privilege of being involved in the whole spectrum of care that affects the lives of many people at their most difficult times - everything from delivery of new life to provision of comfort at life's end. Finally, I have greatly enjoyed the opportunity to work with and learn from some of the most talented healthcare professionals, scientists, and educators.

Anesthesia has aspects to attract and sustain all personality types; however, it is crucial to develop the know-how to work both as a team player and as a team leader, to keep a cool head during crises, and to remain vigilant at all other times. On first inspection, the specialty of anesthesia appears to limit our patient involvement by placing us predominantly in the operating room where we have restricted interpersonal contact with patients. In fact, the opposite is true. We often have direct contact with patients during their most stressful times. During these moments, we must combine generous amounts of compassion and concentrated interpersonal skills. In addition, I have learned over the years that a competent anesthesiologist must acquire a thorough knowledge of many aspects of medicine, surgery, critical care, and obstetrics. The chronic pain specialist will also require skills in counselling and psychotherapy. In summary, the standard reasons students give for choosing to apply to an anesthesia program ${ }^{5,6}$ form only a small part of the varied roles of the anesthesiologist.

For all the above reasons, my recommendation would be for students with an interest in anesthesia to spend significant time learning more about the specialty. A passion for supporting and improving patient care is vital, and while other motivations, such as remuneration and location of practice, may seem important at the end of medical school, these incentives may not sustain one's passion for ten or 20 years. In anesthesia, we want to attract and mentor future leaders in medicine, and passion for the nature of the work will form the foundation of future success.

Positive role models have been shown to attract candidates to anesthesia. ${ }^{6}$ Nevertheless, once a mentor is appointed, further mentorship is vital in order to continue developing the competencies of those with an interest in leadership in clinical care, education, or research. In their editorial in this issue of the Journal, O'Leary and Murphy ${ }^{2}$ discuss the importance of mentoring future leaders in anesthesia, but they do not define the process towards achieving success. The consistent message I have grasped over my career is that successful mentorship is determined by identifying individuals who have a passion for influencing positive change and then supporting them with the time and guidance they need to achieve their goals. ${ }^{\mathrm{B}}$

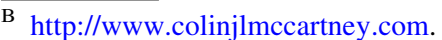

The specialty of anesthesia has a strong tradition of developing physician leaders. Some of the most highly respected physicians in the last 200 years have also been anesthesiologists. Individuals such as John Snow, Virginia Apgar, Harold Griffith, and John Bonica (to name a few) were all anesthesiologists who changed the practice of medicine through their innovation and leadership.

There are many reasons why so many anesthesiologists become leaders, ${ }^{\mathrm{B}}$ but I would highlight three key factors: First, anesthesia is a holistic specialty. Since we care for all patients throughout many different specialties and have sound knowledge of how patients are cared for across the hospital system, we typically take a holistic view of patient care. Second, the anesthesiologist needs to be a team player to work successfully with the perioperative team and often becomes a leader within that group. Accordingly, anesthesiologists are usually skilled at balancing the concerns of several different professions and focusing these on developing best patient care. Finally, we usually work in all areas of the hospital and develop strong collaborative relationships with all specialties, including family medicine -again related to the specialty's holistic nature.

Although we are fortunate to attract some of the best and brightest medical students to our specialty, we must strive to mentor these individuals in order to help them become the next generation of leaders in patient care, education, and research. At the completion of medical school, the choice of specialty can be daunting for many students, but those who have enjoyed their experience in anesthesia should not hesitate to apply. The specialty of anesthesia has something for all whether your interests lie in critical care, pain medicine, obstetrics, or in another of the many subspecialties. Even after 22 years, I still derive great satisfaction from caring for the sickest patients or those with severe pain. It is truly a privilege to have a job that is tremendously rewarding and allows me to connect with so many interesting and inspiring people. The specialty of anesthesia needs great doctors who are willing to commit their careers to caring for people, teaching the next generation, and investigating the best ways to care for our patients. "So......why do you want to be an anesthesiologist?" Here is the simplest answer to the question: The practice of anesthesia is the practice of medicine. That is why I chose it.

\section{Alors... pourquoi voulez-vous devenir anesthésiologiste?}

« Choisis un métier qui te plaît et tu n'auras pas à travailler un seul jour de ta - Confucius

Chaque année, pendant le processus de candidature et de sélection du Service canadien de jumelage des résidents 
pour les postes de formation en anesthésie, on pose une question bien connue aux étudiants en médecine: «Pourquoi voulez-vous devenir anesthésiologiste? » Bon nombre des étudiants de médecine n'auront été que très peu exposés à l'anesthésie clinique et cette question sera difficile. La plupart des candidats ne sauront probablement pas réellement à quoi ressemble la pratique d'un anesthésiologiste.

Dans ce numéro du Journal, plusieurs articles explorent ce thème. Selon Khan et coll. ${ }^{1}$ (et O'Leary et Murphy ${ }^{2}$ dans leur éditorial d'accompagnement), le fait de trouver un domaine qui titille « l'esprit » et d'avoir le bon mentor (qu'il s'agisse d'un mentorat traditionnel ou d'un mentorat 'entre pairs') sont parmi les facteurs qui influencent le choix d'une carrière en anesthésie. A Étant donné l'importance reconnue du mentorat, Zakus et coll. ${ }^{4}$ vont plus loin encore en examinant si nos programmes de résidence proposent des occasions formelles de mentorat et observent, sans surprise peut-être, que l'existence d'un programme formel de mentorat est associée à une plus forte probabilité de recevoir du soutien. Pour la plupart des étudiants, les principaux facteurs influençant le choix de l'anesthésie comme carrière sont les défis que représentent la prise en charge de patients en état critique, l'application clinique de la physiologie et de la pharmacologie, la nature «pratique » de la spécialité ainsi que l'influence de modèles positifs. ${ }^{5-7}$

Personnellement, l'un des facteurs les plus déterminants dans mon choix de carrière a été d'avoir des modèles qui ont pris le temps de me montrer les talents spécifiques aux anesthésiologistes. Après ma médecine, j'ai passé ma première année dans deux grands hôpitaux communautaires où j'avais la charge de plusieurs patients gravement malades et de patients souffrant de douleurs non contrôlées, tant dans les services de médecine que de chirurgie. Lors de la prise en charge de ces patients, j'ai rapidement réalisé que bien souvent on appelait l'anesthésiologiste pour prendre en charge les situations critiques lorsque les autres spécialistes ne savaient plus que faire. Les compétences et la compassion de ces collègues, mais aussi leur patience et leur calme - voilà ce qui m'a inspiré. À la fin de l'année, j'avais décidé de devenir anesthésiologiste et c'est l'influence de ces futurs collègues charismatiques qui m'a tourné vers cette spécialité. Bon nombre de mes collègues ont eu des expériences semblables ${ }^{6}$ mais, comme plusieurs étudiants en fin de médecine, lorsque j'ai choisi l'anesthésie comme spécialité, je n'avais qu'une vague idée de ce à quoi la pratique ressemblait vraiment.

Aujourd'hui, 22 ans plus tard, et en qualité de chef de l'un des plus grands départements d'anesthésie au Canada, je pense que je commence enfin à comprendre ce que c'est que d'être un anesthésiologiste!
Anesthésie: voici un bien petit mot pour décrire l'une des spécialités médicales les plus holistiques, qui recoupe une exposition à la quasi-totalité des domaines de l'hôpital, y compris bien sûr les compétences périopératoires évidentes, mais également une expertise spécifique dans la prise en charge des patients en état critique ou souffrant de douleurs réfractaires aiguës, chroniques, et bien souvent liées à un cancer. Au cours de ces décennies de formation et de pratique, j'ai eu le privilège de toucher à tous les aspects des soins, allant de la venue au monde d'une nouvelle vie à l'offre de confort en fin de vie. J'ai eu le privilège d'être impliqué dans les vies de nombreuses personnes au moment où ces vies étaient au plus difficile. Enfin, j'ai eu l'occasion de travailler avec et d'apprendre de certains des professionnels de la santé, des scientifiques et des formateurs les plus talentueux qui soient.

L'anesthésie a quelque chose à offrir pour séduire et nourrir tous les types de personnalité. Ceci étant dit, certaines qualités sont absolument cruciales: la capacité à travailler en équipe et à mener une équipe, à garder la tête froide en temps de crise et à être vigilant en tout temps. À première vue, la spécialité de l'anesthésie semble limiter notre implication auprès des patients en nous plaçant principalement en salle d'opération, là où nos contacts avec le patient sont restreints. En fait, c'est tout le contraire: nous sommes en contact direct avec le patient en ses moments les plus stressants, c'est pourquoi il nous faut incorporer une bonne dose de compassion et des qualités interpersonnelles concentrées dans notre pratique. De plus, j'ai réalisé au fil des années qu'afin d'être un anesthésiologiste compétent, il fallait posséder une connaissance approfondie de plusieurs aspects de la médecine, de la chirurgie, des soins critiques et de l'obstétrique. Le spécialiste de la douleur chronique devra également posséder des compétences en counseling et en psychothérapie. En somme, les raisons usuelles qui poussent les étudiants à postuler en anesthésie ${ }^{5,6}$ ne forment qu'une infime portion des divers rôles de l'anesthésiologiste.

C'est pourquoi je recommande à tous les étudiants qui s'intéressent à l'anesthésie de consacrer du temps à en savoir plus sur la spécialité. Le soutien et l'amélioration des soins aux patients doivent être une passion absolue. Les autres motivations, telles que la rémunération ou le lieu de pratique, peuvent sembler bien importantes à la fin de l'école de médecine; mais ces motivations ne soutiendront pas une passion dans dix ou vingt ans. En anesthésie, nous souhaitons attirer et former les futurs chefs de file de la médecine: une passion pour la nature de la tâche est nécessaire pour poser les fondations d'un succès futur.

Il a été démontré que les modèles positifs attiraient les candidats en anesthésie. ${ }^{6}$ Toutefois, une fois en poste, le mentorat futur est essentiel afin de poursuivre l'épanouissement des individus intéressés par le 
leadership en matière de soins cliniques, d'éducation ou de recherche. Dans leur éditorial dans ce numéro, O'Leary et Murphy $^{2}$ traitent de l'importance de guider les futurs chefs de file de l'anesthésie, mais ils ne définissent pas le processus pour y parvenir. Le message constant que j'ai reçu tout au long de ma carrière est que pour réussir, il faut identifier les individus qui arborent une passion pour le changement positif, puis les soutenant en leur accordant le temps et le mentorat nécessaires pour atteindre leurs objectifs. $^{\mathrm{B}}$

La spécialité de l'anesthésie peut se targuer d'être un des berceaux traditionnels pour la formation de chefs de file en médecine. Certains des médecins les plus respectés des 200 dernières années étaient également des anesthésiologistes. Des individus tels que John Snow, Virginia Apgar, Harold Griffith et John Bonica, pour ne nommer qu'eux, étaient tous des anesthésiologistes qui ont transformé la pratique de la médecine grâce à leur innovation et à leur leadership.

Les raisons expliquant pourquoi il y a tant d'anesthésiologistes qui deviennent des chefs de file sont nombreuses $;{ }^{\mathrm{B}} \mathrm{j}$ 'aimerais en souligner trois. Premièrement, l'anesthésie est une spécialité holistique, qui prend soin de tous les patients dans différentes spécialités; nous avons généralement une vue holistique des soins aux patients et une solide connaissance de la façon dont les patients sont pris en charge dans le système hospitalier. Deuxièmement, l'anesthésiologiste doit avoir l'esprit d'équipe afin de bien travailler avec l'équipe périopératoire, et il devient souvent un leader au sein de ce groupe. Par conséquent, les anesthésiologistes savent en général comment tenir compte des préoccupations de plusieurs professions différentes et comment orienter ces préoccupations pour mettre au point de meilleurs soins aux patients. Enfin, et une fois de plus en lien avec la nature holistique de notre spécialité, nous travaillons en général dans tout l'hôpital et développons des relations de collaboration durables avec toutes les spécialités, y compris la médecine familiale.

Bien que nous ayons la chance d'attirer certains des étudiants en médecine les plus brillants, nous devons nous efforcer de guider ces personnes afin qu'elles forment la prochaine génération de chefs de file en matière de soins aux patients, d'éducation et de recherche. À la fin de l'école de médecine, le choix d'une spécialité peut être ardu pour bon nombre d'étudiants; mais ceux qui ont aimé leur expérience en anesthésie ne devraient pas hésiter à postuler. La spécialité de l'anesthésie a quelque chose à offrir à chacun, que vous soyez intéressé par les soins critiques, la médecine de la douleur, l'obstétrique ou l'une des nombreuses autres surspécialités. Le fait de prendre soin des patients les plus malades ou de ceux souffrant de douleurs graves me satisfait encore, même après 22 ans. C'est véritablement un privilège que d'avoir un métier si gratifiant, et qui me permet de côtoyer tant de personnes intéressantes et inspirantes. La spécialité de l'anesthésie a besoin de grands médecins qui sont prêts à engager leur carrière à prendre soin des gens, à enseigner à la prochaine génération, et à chercher la meilleure façon de prendre soin de nos patients. Pour répondre à la question « Pourquoi voulez-vous devenir anesthésiologiste? », la réponse la plus simple est que la pratique de l'anesthésie est la pratique de la médecine. Voilà pourquoi je la choisis.

Competing interests None declared.

Conflits d'intérêt aucun.

\section{References}

1. Khan J, Gilbert J, Sharma A, LeManach Y, Yee D. Perspectives of anesthesia residents training in Canada on fellowship training, research and future practice location. Can J Anesth 2015; 62: this issue. DOI: $10.1007 / \mathrm{s} 12630-015-0420-1$.

2. O'Leary S, Murphy M. Anesthesiology: Is it a career, a job or both? Can J Anesth 2015; 62: this issue. DOI: 10.1007/s12630015-0421-0.

3. Miller DR, McCartney CJ. Mentoring during anesthesia residency training: challenges and opportunities. Can J Anesth 2015; 62: this issue. DOI: $10.1007 / \mathrm{s} 12630-015-0419-7$.

4. Zakus $P$, Gelb AW, Flexman AM. A survey of mentorship among Canadian anesthesiology residents. Can J Anesth. 2015: 62: this issue. DOI: 10.1007/s12630-015-0418-8.

5. Khan FA, Hamdani GA. Factors influencing the choice of anesthesia as a career in a developing country. Middle East $\mathbf{J}$ Anaesthesiol 2007; 19: 149-57.

6. Roberts LJ, Khursandi DC. Career choice influences in Australian anaesthetists. Anaesth Intensive Care 2002; 30: 355-9.

7. Wass CT, Long TR, Randle DW, Rose SH, Faust RJ, Decker PA. Recruitment of house staff into anesthesiology: a re-evaluation of factors responsible for house staff selecting anesthesiology as a career and individual training program. J Clin Anesth 2003; 15: 289-94. 